\title{
The therapeutic potential of resistant starch in modulation of insulin resistance, endotoxemia, oxidative stress and antioxidant biomarkers in women with type 2 diabetes: a randomized controlled clinical trial
}

\begin{abstract}
Aims: This trial aims to determine the effects of resistant starch (RS) subtype 2 (RS2) on glycemic status, metabolic endotoxemia and markers of oxidative stress. Methods: A randomized, controlled, parallel-group clinical trial group of 56 females with type 2 diabetes mellitus (T2DM) was divided to 2 groups. The intervention group $(\mathrm{n}=28)$ and control group $(\mathrm{n}=28)$ received $10 \mathrm{~g} /$ day $\mathrm{RS} 2$ or placebo for 8 weeks, respectively. Fasting blood samples were taken to determine glycemic status, endotoxin, high sensitivity C-reactive protein (hsCRP), malondialdehyde (MDA), total antioxidant capacity (TAC), antioxidant enzymes concentrations as well as uric acid at baseline and after the intervention. Results: After 8 weeks, RS2 caused a significant decrease in the levels of MDA (-34.10\%), glycosylated hemoglobin $(-9.40 \%)$, insulin $(-29.36 \%)$, homeostasis model of insulin resistance $(-32.85 \%)$ and endotoxin (-25.00\%), a significant increase in TAC (18.10\%) and glutathione peroxidase $(11.60 \%)$ as compared with control. No significant changes were observed in fasting plasma glucose, quantitative insulin sensitivity check index, hs-CRP, superoxide dismutase, catalase and uric acid in the RS2 group as compared with the control group. Conclusion: Supplementation with RS2 may be improved glycemic status, endotoxemia and markers of oxidative stress in patients with T2DM.
\end{abstract}

Keyword: Antioxidant; Endotoxemia; Insulin; Malondialdehde; Resistant starch; Type 2 diabetes 